\title{
Pleural Epithelioid Hemangioendothelioma: Literature Summary and Novel Case Report
}

\author{
Julita Salijevska ${ }^{\mathrm{a}, \mathrm{d}}$, Robert Watson ${ }^{\mathrm{a}}$, Amy Clifford ${ }^{\mathrm{b}}$, Andrew I. Ritchie ${ }^{\mathrm{b}}$, Francisco Mauric ${ }^{\mathrm{c}}$, \\ David Adeboyeku ${ }^{\mathrm{b}}$
}

\begin{abstract}
Epithelioid hemangioendothelioma (EHE) is a rare malignant cancer of vascular origin that can affect multiple and varied tissue sites. A subtype of EHE, pulmonary epithelioid hemangioendothelioma (PHE), is more unusual with only 200 reported cases. Of these, only 27 have been classified as pleural in origin. Based on available literature, the average age of presentation of pleural PHE is 45.7 years with a male preponderance of 2.375. A summary of all published case reports reveals significant heterogeneity both in presentation and management. Here we add to this knowledge-base with a report of an unusual case of pleural PHE in a 36-year-old female who presented with a 6 -week history of chest pain and breathlessness. Significant challenges in the diagnosis and management of patients with pleural PHE exist, including a wide initial differential diagnosis and difficulties in obtaining tissue specimens, coupled with relatively limited treatment options. Early referral to a cardiothoracic center for videoassisted thoracoscopic biopsy is crucial in facilitating a diagnosis and allowing adequate pleural drainage for symptomatic relief.
\end{abstract}

Keywords: Epithelioid hemangioendothelioma; Pleura; Pulmonary hemangioendothelioma; Intravascular bronchoalveolar tumor

\section{Introduction}

Epithelioid hemangioendothelioma (EHE) is a rare malignant cancer of vascular origin. Cases have been reported affecting bone, brain, the meninges, soft tissue, gastrointestinal tract,

Manuscript accepted for publication April 14, 2015

amperial College Healthcare NHS Trust, Charing Cross Hospital, Fulham Palace Road, London W6 8RF, UK

${ }^{b}$ The North West London Hospitals NHS Trust, Northwick Park Hospital, Watford Road, Harrow, Middlesex, HA1 3UJ, UK

'Imperial College Healthcare NHS Trust, The Hammersmith Hospital, Du Cane Road, London W12 0HS, UK

${ }^{\mathrm{d} C o r r e s p o n d i n g ~ A u t h o r: ~ J u l i t a ~ S a l i j e v s k a, ~ I m p e r i a l ~ C o l l e g e ~ H e a l t h c a r e ~ N H S ~}$ Trust, Charing Cross Hospital, Fulham Palace Road, London W6 8RF, UK. Email: julita.salijevska@imperial.nhs.uk

doi: http://dx.doi.org/10.14740/jocmr2174w mediastinum, spleen, breast, testicle and skin with a variable grade of malignancy [1,2]. It typically follows an intermediate course between hemangioma and conventional angiosarcoma [3-5]; however, the pleural variant of the disease is less frequently described and is known to follow a more aggressive clinical course [2].

Pulmonary epithelioid hemangioendothelioma (PHE) was first described in 1975 by Dail and Leibow [2]. However, due to its low incidence, the true epidemiology of PHE remains unknown. To our knowledge, there have been less than 200 cases of PHE reported in the literature with only 27 cases further classified as pleural epithelioid hemangioendothelioma, as shown in Table 1 [1, 6-21].

Analysis of these cases reveals significant heterogeneity in both presentation and management (Table 1). The mean age at presentation is 45.7 years with a female/male ratio of 1:2.375 and a mean survival of 9.6 months. There was a heterogenous array of symptoms at presentation; however, chest pain was a consistent feature, along with dyspnea and cough. No consistent management approach was used with some patients undergoing surgery, some receiving chemotherapy, some a combination and some no specific treatment. Of the patients that received chemotherapy a variety of regimens were used; however, carboplatin and etoposide was the most popular with six out of 11 patients receiving this combination of treatment.

Here we describe a more unusual case of pleural PHE in a young female presenting with a 6-week history of right-sided chest pain, found to have a complete whiteout on chest radiograph secondary to a malignant pleural effusion. This adds further to the limited published knowledge of pleural PHE and gives further insight into the investigation and management options.

\section{Case Report}

A 36-year-old Caucasian female attended the accident and emergency department with right-sided chest pain. The pain was described as dull and aching, with a pleuritic nature. It was sited diffusely over the right anterior and lateral chest wall and was reproducible on palpation. The pain had increased in severity over the preceding 6 weeks and at the time of admission was associated with mild dyspnea. Prior to presentation, the patient reported no weight loss, fevers or night sweats. Her family history and past medical history were unremarkable. 


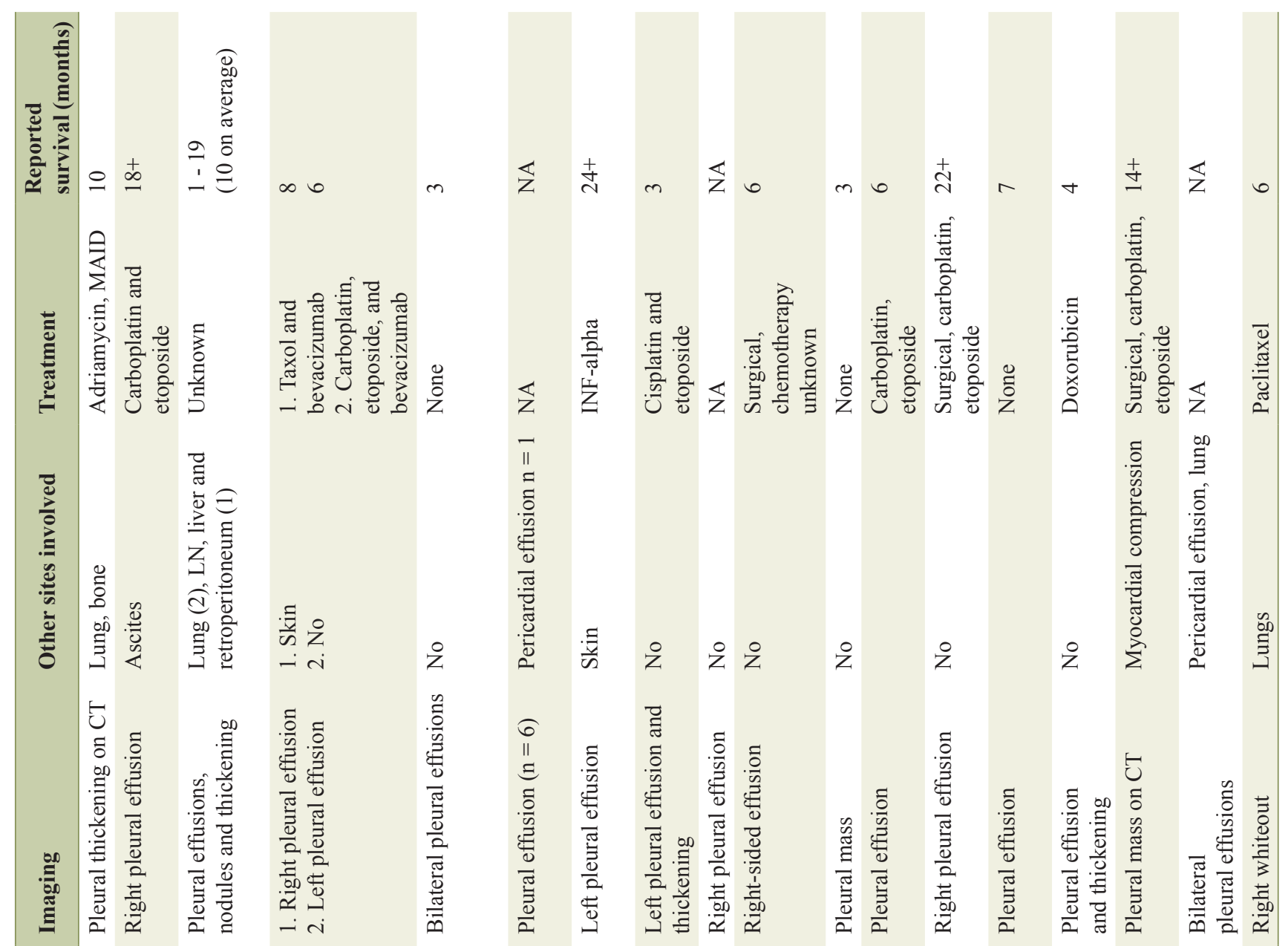

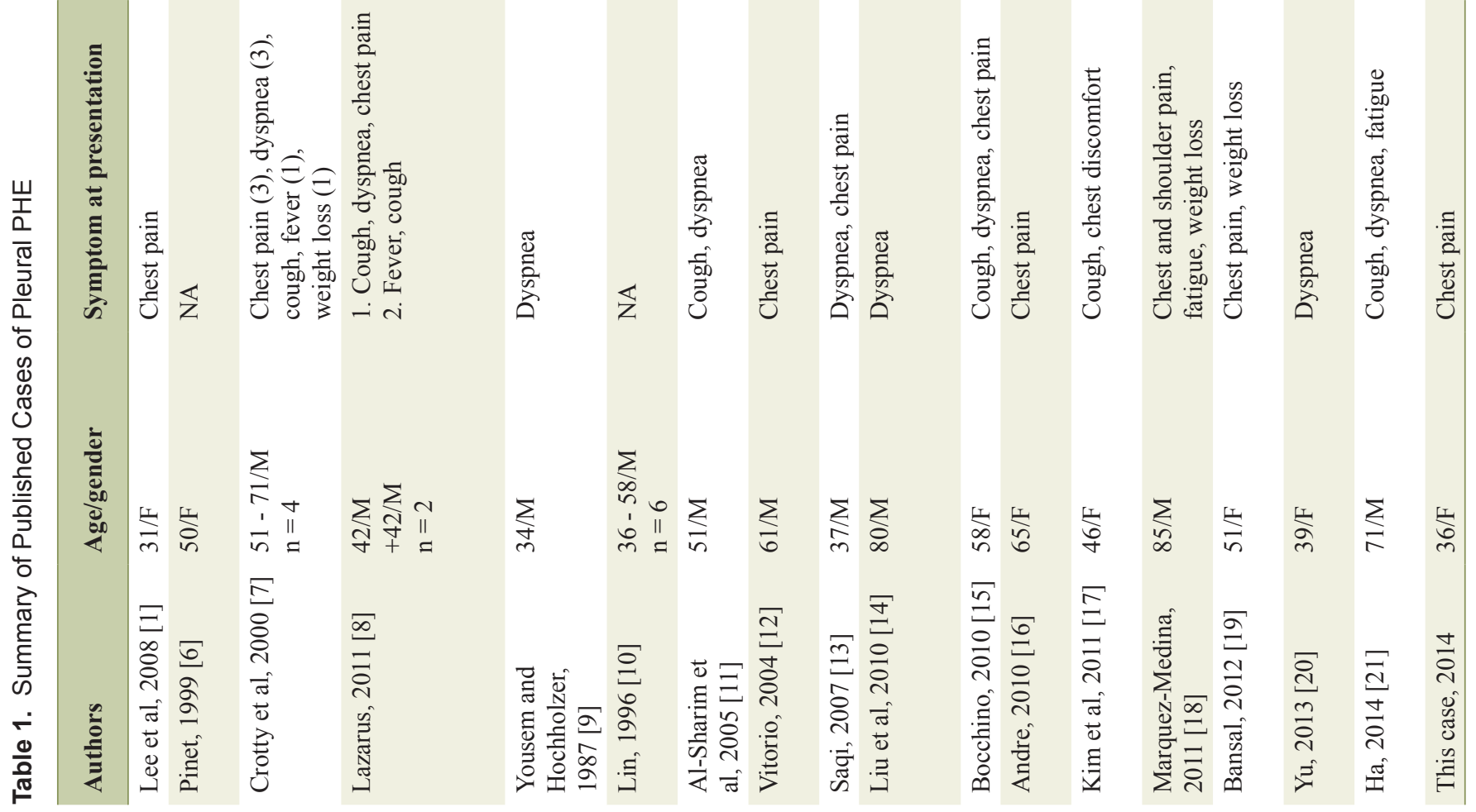




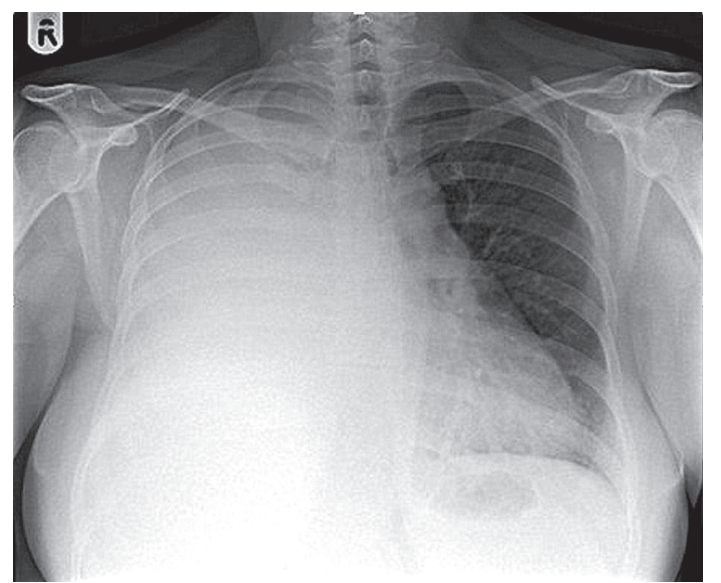

Figure 1. Chest radiograph demonstrating complete opacification of the right hemithorax with mild mediastinal shift to the left side.

However, she was a cigarette smoker (10 pack-year history).

On examination, the patient was pale and tachypnoeic. Her observations demonstrated a borderline tachycardia at 92 beats per minute and pulse oximetry of $92 \%$ on room air. On examination of the respiratory system, there was decreased expansion, dullness to percussion and decreased breath sounds over the right hemithorax. There was no jaundice, digital clubbing, skin changes or palpable lymphadenopathy. The breast examination was unremarkable.

Initial laboratory findings demonstrated a leukocytosis (white blood cells $18.6 \times 10^{9} / \mathrm{L}$ ) and neutrophilia (neutrophils $15.9 \times 10^{9} / \mathrm{L}$ ), anemia (hemoglobin $9.8 \mathrm{~g} / \mathrm{dL}$ ), thrombocytosis (platelets $518 \times 10^{9} / \mathrm{L}$ ), hypercalcemia (corrected calcium 2.81 $\mathrm{mmol} / \mathrm{L})$, and raised C-reactive protein $(117 \mathrm{mg} / \mathrm{L})$.

A chest radiograph revealed complete opacification of the right hemithorax with mild mediastinal shift to the left side (Fig. 1). This was further characterized by a contrast enhanced computed tomography (CT) scan of the thorax, abdomen and pelvis which showed a large loculated right pleural effusion with associated right lung collapse, significant multifocal right-sided pleural thickening, and multiple pulmonary nodules on the left. No abnormalities were seen below the diaphragm, save for

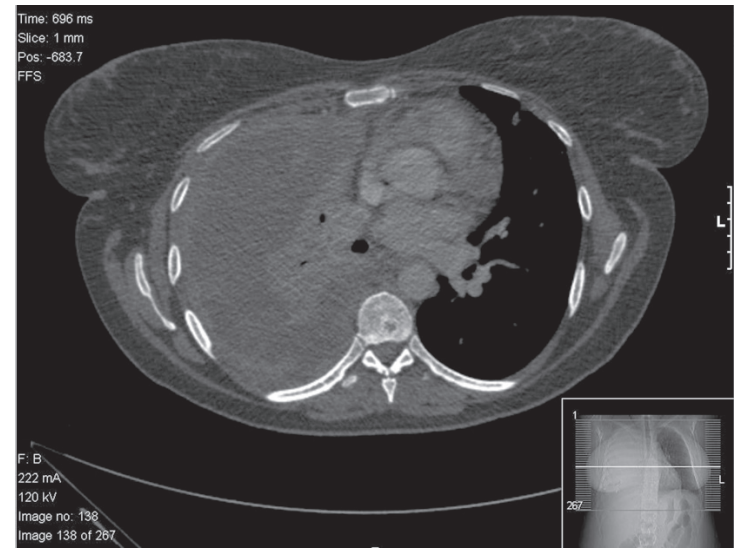

Figure 2. CT scan demonstrating pleural thickening, effusion, right lung collapse and left mediastinal shift.

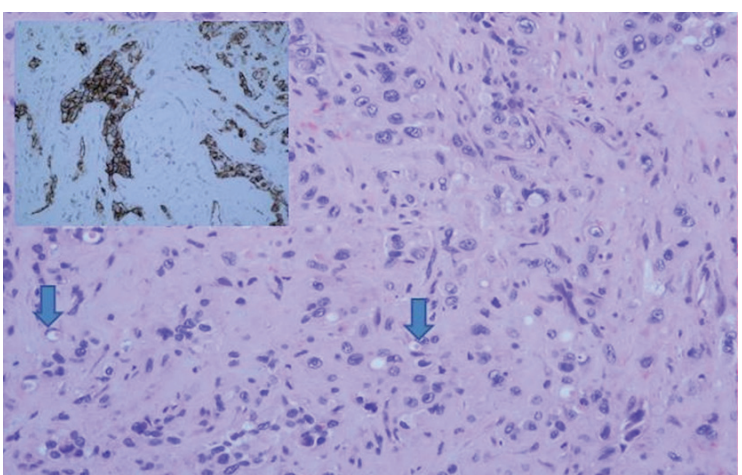

Figure 3. Histology from VATS guided pleural biopsy. Tumor shows cords, nests and groups of medium or large sized epithelioid cells with inconspicuous nucleoli set in a myxoid stroma. Some cells show mild atypia. Many cells have intracytoplasmic lumina which occasionally contain red blood cells (arrows). Tumor cells are positive for CD31 (insert) and CD34.

some simple renal cysts. Appearances were reported to be likely in keeping with pleural and pulmonary metastases (Fig. 2).

The patient went on to have an ultrasound guided Seldinger chest drain inserted. Seven hundred twenty milliliters of heavily blood stained pleural fluid drained and was sent for analysis: $\mathrm{pH}$ 6.99, protein $52 \mathrm{~g} / \mathrm{L}$, and LDH $642 \mathrm{IU} / \mathrm{L}$. Cytological analysis did not detect any malignant cells. The microscopy and subsequent culturing was unremarkable.

Considering the patient's young age and the radiological findings, our provisional diagnosis was malignancy with a likely primary of breast, ovary, lymphoma or lung. With this in mind our patient went on to have an ultrasound examination of her breasts, which revealed some areas of benign breast changes but no focal suspicious appearances. Transvaginal ultrasound demonstrated an anteverted uterus, endometrial thickness of $5 \mathrm{~mm}$ and normal ovaries.

Despite chest drain insertion and the initial volume drained, we were subsequently unable to remove a significant volume of pleural fluid from the right hemi-thorax. The patient was referred to a tertiary center with cardiothoracic facilities where video-assisted thoracoscopic surgery (VATS) was performed. This allowed successful evacuation of the septated pleural effusion and the opportunity to perform pleural biopsies.

The histological diagnosis was confirmed by both conventional examination demonstrating the characteristic tumor cells with cords and nests, and immunohistochemistry with expression of MNF116 and CK7, D2-40, CD31 and CD34 antibodies (Fig. 3).

Post-operatively our patient recovered well and was referred for specialist oncological input which she received at a quaternary London cancer center. Although an effective treatment for pleural PHE has yet to be established, Pinet et al [6] have reported the case of an aggressive pleural PHE which had a complete response to treatment with carboplatin and etoposide. Our patient received weekly cycles of paclitaxel, a mitotic inhibitor, which has also demonstrated a clinical response in case reports [22], for a 3-month period. Unfortunately, during this time she required frequent admissions for pain control and sadly passed away 6 months after her diagnosis. 


\section{Discussion}

Pleural EHE is an extremely rare entity. Detecting it in the early stages is often not possible, as initial symptoms are typically non-specific with chest pain, dyspnea or persistent cough, the most frequently reported presenting complaint [7, 8]. Furthermore literature review has highlighted individual cases of incidental diagnosis in asymptomatic patients [23].

Our case highlights the aggressive nature and late presentation of this malignancy even in a young patient with good pre-morbid baseline functioning. It also demonstrates the need for a systematic approach to all patients presenting with a unilateral pleural effusion of likely malignant origin as more common etiologies need to be excluded prior to referral to a specialist oncology center. In such scenarios, the clinical team should move quickly to establish a diagnosis so that treatment can be started as soon as possible, especially given the poor life expectancy associated with this cancer.

It is worth bearing in mind that pleural PHE can lead to heavily loculated effusions. Therefore VATS can play a vital role in securing a tissue diagnosis and evacuating the pleural effusion when less invasive methods fail.

As with our patient, pleuritic pain is a dominant feature of pleural PHE. Early referral to an acute pain service must be considered in patients that do not respond to conventional analgesia [24] as poorly controlled symptoms may lead to a delay in oncology referral and importantly a delay in starting chemotherapy and radiotherapy.

Further research and case reporting are required to contribute to the data regarding the clinical course and the natural history of this rare malignancy in order to build an evidence base for treatment. A successful and accepted chemotherapeutic treatment regime is yet to be established and adopted with a range of different options currently employed.

\section{Conclusion}

Whilst EHE is an extremely rare malignant cancer, and its pleural subtype is even rarer, it is important we continue to characterize its clinical course and response to treatments. Early cardiothoracic input to allow for adequate pleural drainage and biopsy along with aggressive pain management are important components of the initial management. A standard chemotherapeutic regime is yet to be widely adopted and further work in this area is needed.

\section{Competing Interests}

The authors declare that they have no competing interests.

\section{Funding}

The authors confirm that the research was conducted in the absence of any study sponsor.

\section{References}

1. Lee YJ, Chung MJ, Jeong KC, Hahn CH, Hong KP, Kim YJ, Kim YT. Pleural epithelioid hemangioendothelioma. Yonsei Med J. 2008;49(6):1036-1040.

2. Dail DH, Liebow AA, Gmelich JT, Friedman PJ, Miyai K, Myer W, Patterson SD, et al. Intravascular, bronchiolar, and alveolar tumor of the lung (IVBAT). An analysis of twenty cases of a peculiar sclerosing endothelial tumor. Cancer. 1983;51(3):452-464.

3. Kitaichi M, Nagai S, Nishimura K, Itoh H, Asamoto H, Izumi T, Dail DH. Pulmonary epithelioid haemangioendothelioma in 21 patients, including three with partial spontaneous regression. Eur Respir J. 1998;12(1):89-96.

4. Weiss SW, Ishak KG, Dail DH, Sweet DE, Enzinger FM. Epithelioid hemangioendothelioma and related lesions. Semin Diagn Pathol. 1986;3(4):259-287.

5. Bollinger BK, Laskin WB, Knight CB. Epithelioid hemangioendothelioma with multiple site involvement. Literature review and observations. Cancer. 1994;73(3):610615.

6. Pinet C, Magnan A, Garbe L, Payan MJ, Vervloet D. Aggressive form of pleural epithelioid haemangioendothelioma: complete response after chemotherapy. Eur Respir J. 1999; 14(1):237-238.

7. Crotty EJ, McAdams HP, Erasmus JJ, Sporn TA, Roggli VL. Epithelioid hemangioendothelioma of the pleura: clinical and radiologic features. AJR Am J Roentgenol. 2000;175(6):1545-1549.

8. Lazarus A, Fuhrer G, Malekiani C, McKay S, Thurber J. Primary pleural epithelioid hemangioendothelioma (EHE)--two cases and review of the literature. Clin Respir J. 2011;5(1):e1-5.

9. Yousem SA, Hochholzer L. Unusual thoracic manifestations of epithelioid hemangioendothelioma. Arch Pathol Lab Med. 1987;111(5):459-463.

10. Lin BT, Colby T, Gown AM, Hammar SP, Mertens RB, Churg A, Battifora H. Malignant vascular tumors of the serous membranes mimicking mesothelioma. A report of 14 cases. Am J Surg Pathol. 1996;20(12):1431-1439.

11. Al-Shraim M, Mahboub B, Neligan PC, Chamberlain D, Ghazarian D. Primary pleural epithelioid haemangioendothelioma with metastases to the skin. A case report and literature review. J Clin Pathol. 2005;58(1):107-109.

12. Vitorio; Epithelioid hemangioendothelioma of the pleura. J Bras Pneumol. 2004;30(1).

13. Saqi A, Nisbet L, Gagneja P, Leslie KO. Primary pleural epithelioid hemangioendothelioma with rhabdoid phenotype: report and review of the literature. Diagn Cytopathol. 2007;35(4):203-208.

14. Liu JX, Shiau MC, Nonaka D. An 80-year-old man with shortness of breath and large right-sided pleural effusion. Chest. 2010;138(5):1247-1252.

15. Bocchino M, Barra E, Lassandro F, Ranieri F, Muto R, Rea G. Primary pleural haemangioendothelioma in an Italian female patient: a case report and review of the literature. Monaldi Arch Chest Dis. 2010;73(3):135-139.

16. Andre ST, Valente C, Paiva B, Pego A, Carvalho L, Luis 
AS. [Epithelioid hemangioendothelioma of the pleura - A rare presentation of a clinical case]. Rev Port Pneumol. 2010;16(3):477-482.

17. Kim EA, Lele SM, Lackner RP. Primary pleural epithelioid hemangioendothelioma. Ann Thorac Surg. 2011;91(1):301-302.

18. Marquez-Medina D, Samame-Perezvargas JC, Tuset-DerAbrain N, Montero-Fernandez A, Taberner-Bonastre T, Porcel JM. Pleural epithelioid hemangioendothelioma in an elderly patient. A case report and review of the literature. Lung Cancer. 2011;73(1):116-119.

19. Bansal A, Chawla M, Cohen PJ, Kwon JS. Pleural epithelioid hemangioendothelioma. Lung. 2012;190(4):469-470.

20. Yu L, Gu T, Xiu Z, Shi E, Zhao X. Primary pleural epi- thelioid hemangioendothelioma compressing the myocardium. J Card Surg. 2013;28(3):266-268.

21. Ha SY, Choi IH, Han J, Choi YL, Cho JH, Lee KJ, Sun JM. Pleural epithelioid hemangioendothelioma harboring CAMTA1 rearrangement. Lung Cancer. 2014;83(3):411415.

22. Gaur S, Torabi A, O'Neill TJ. Activity of angiogenesis inhibitors in metastatic epithelioid hemangioendothelioma: a case report. Cancer Biol Med. 2012;9(2):133-136.

23. Syred KS, Gibbs AR, Attanoos RL. University Hospital Llandough, Cardiff. Pleural angiosarcomas: pitfalls for the pathologist. Cardiff Pathology. 2009:55.

24. World Health Organization. Cancer pain relief. With a guide to opioid availability (2 ed.). Geneva: WHO.1996. 